\title{
A conjecture of Beauville and Catanese revisited
}

\section{Journal Article}

\section{Author(s):}

Pink, Richard; Roessler, Damian

Publication date:

2004

Permanent link:

https://doi.org/10.3929/ethz-b-000160331

\section{Rights / license:}

In Copyright - Non-Commercial Use Permitted

\section{Originally published in:}

Mathematische Annalen 330(2), https://doi.org/10.1007/s00208-004-0549-7 


\title{
A conjecture of Beauville and Catanese revisited
}

\section{Richard Pink • Damian Roessler}

Received: 30 November 2003 / Published online: 23 June 2004 - ㅇ Springer-Verlag 2004

\begin{abstract}
A theorem of Green, Lazarsfeld and Simpson (formerly a conjecture of Beauville and Catanese) states that certain naturally defined subvarieties of the Picard variety of a smooth projective complex variety are unions of translates of abelian subvarieties by torsion points. Their proof uses analytic methods. We refine and give a completely new proof of their result. Our proof combines galois-theoretic methods and algebraic geometry in positive characteristic. When the variety has a model over a function field and its Picard variety has no isotrivial factors, we show how to replace the galois-theoretic results we need by results from model theory (mathematical logic). Furthermore, we prove partial analogs of the conjecture of Beauville and Catanese in positive characteristic.
\end{abstract}

\section{Introduction}

In this article we shall refine and give a new proof of the following result of Green, Lazarsfeld and Simpson, which was conjectured by Beauville and Catanese. To formulate it, let $Y$ be a smooth projective complex manifold and let $\operatorname{Pic}^{0}(Y)$ be its Picard variety, which classifies the holomorphic line bundles on $Y$ which are algebraically equivalent to 0 . For integers $i, j, m \geq 0$ let

$$
S_{m}^{i, j}(Y):=\left\{\mathcal{L} \in \operatorname{Pic}^{0}(Y)(\mathbb{C}) \mid \operatorname{dim}_{\mathbb{C}}\left(H^{i}\left(Y, \Omega_{Y}^{j} \otimes \mathcal{L}\right)\right) \geq m\right\}
$$

The semi-continuity theorem implies that this set is Zariski-closed.

Theorem 1.1 (Green-Lazarsfeld; Simpson). The irreducible components of $S_{m}^{i, j}(Y)$ are translates of abelian subvarieties of $\operatorname{Pic}^{0}(Y)$ by torsion points.

In [5], Green and Lazarsfeld prove that the irreducible components of $S_{m}^{i, j}(Y)$ are translates of abelian subvarieties of $\operatorname{Pic}^{0}(Y)$ by some points. Their proof relies on an in-depth analysis of the relative de Rham complex of the universal family over $\operatorname{Pic}^{0}(Y)$. In [11], Simpson proves the full statement; his proof relies on the fact that the set $S_{m}^{i, j}(Y)$ carries several different algebraic structures, which

R. PINK, D. ROESSLER

Department of Mathematics ETH-Zentrum, CH-8092 Zürich, Switzerland (e-mail: pink@math.ethz.ch, roessler@math.ethz.ch) 
arise from the various descriptions of line bundles that are algebraically equivalent to 0 (e.g. as locally constant systems ofrank 1 or as line bundles with a flat connection). This allows him to reprove the result of Green and Lazarsfeld. To obtain the full Theorem 1.1, he applies a result from transcendance theory, the criterion of Schneider-Lang. Both of the above proofs are analytic in nature. In our proof, we first suppose that $Y$ has a model over a number field. Our proof of the conjecture in this case then relies on a result by Deligne and Illusie (Theorem 3.2) originally used to give a proof of the Kodaira-Nakano vanishing theorem and on a classification theorem (Theorem 2.1). The proof of this classification result combines algebraic geometry in positive characteristic and density arguments. The infinitesimal arguments used in the analytic approach are thus replaced by density arguments in our approach. Finally, one can reduce the general case of the conjecture of Beauville and Catanese to the number field case by standard arguments. The just described proof is contained in Section 3 and the proof of the classification theorem 2.1 is contained in Section 2.

When the variety $Y$ has a model over a function field (in one variable) and $\operatorname{Pic}^{0}(Y)$ has no isotrivial factors (this is made precise in Section 6), we give a proof of Theorem 1.1, which does not use density arguments but relies on results from model-theory (of mathematical logic) instead. We use results of Hrushovski (see [7]) which appear in his proof that the Mordell-Lang conjecture for function fields in characteristic 0 can be deduced from the analogous result in characteristic $p$. This proof is contained in Section 6.

We shall also prove the following result, which improves the result of Simpson. Let $r, k \geq 0$ and let

$$
S_{k}^{r}(Y):=\left\{\mathcal{L} \in \operatorname{Pic}^{0}(Y)(\mathbb{C}) \mid \sum_{i+j=r} \operatorname{dim}_{\mathbb{C}}\left(H^{i}\left(Y, \Omega_{Y}^{j} \otimes \mathcal{L}\right)\right) \geq k\right\} .
$$

Let $c_{r, k} \geq 0$ be the number of irreducible components of $S_{k}^{r}(Y)$. Now let $m \geq 0$ and let $M_{r, m}:=\operatorname{Max}_{k \geq m} c_{r, k}$.

Proposition 1.2. Let $i+j=r$. For each irreducible components $C$ of $S_{m}^{i, j}(Y)$, there exists a torsion point $t \in C$ such that $\varphi(\operatorname{order}(t)) \leq M_{r, m}$.

Here $\varphi$ is Euler's $\varphi$-function $\left(\varphi(a):=\#(\mathbb{Z} / a \mathbb{Z})^{*}\right.$ for all $a \geq 2$ and $\left.\varphi(1)=1\right)$. Since $\varphi(a) \rightarrow \infty$ as $a \rightarrow \infty$, we see that Proposition 1.2 really gives an upper bound for $\operatorname{order}(t)$. We shall give an algebraic proof of this result, although an analytic proof is also possible. The proof is given in Section 3 (Theorem 3.6).

In Section 4, we show the implications of our methods for the natural positive characteristic analog of the conjecture of Beauville and Catanese.

In Section 5, we formulate a conjecture on the cohomology of torsion line bundles, which would imply the natural positive characteristic analog of the conjecture of Beauville and Catanese. 
Acknowledgements. Our thanks go to $\mathrm{H}$. Esnault, who pointed out the conjecture of Beauville and Catanese to the first author and asked whether the techniques of our previous article [10] could be used to tackle this conjecture. We also want to thank C. Soulé for interesting suggestions.

\section{Linear subvarieties of abelian varieties}

In the rest of this article, we shall use the following terminology. If $B$ is an abelian variety defined over an algebraically closed field $L$ and $Z$ is a reduced closed subscheme of $B$, we shall say that $Z$ is linear (resp. completely linear) if the irreducible components of $Z$ are translates of abelian subvarieties of $A$ by some elements of $B(L)$ (resp. some torsion elements of $B(L)$ ). Note that an irreducible completely linear subscheme is also referred to as a torsion subvariety.

If $Y \rightarrow S$ is any morphism of schemes, we shall write $k(s)$ for the residue field of the point $s \in S$ and $Y_{s}$ for $Y \times_{S}$ Spec $k(s)$; we also write $Y_{\bar{s}}$ for $Y \times{ }_{S} \operatorname{Spec} \overline{k(s)}$. If $S \subset \operatorname{Spec} \mathcal{O}_{K}$ for a number field $K$ we also write $Y_{\mathfrak{p}}$ and $Y_{\overline{\mathfrak{p}}}$ for the fibre, respectively the geometric fibre, at a finite prime $\mathfrak{p}$.

The aim of this section is to prove the following classification theorem, that we shall combine with a result a Deligne-Illusie (Theorem 3.2) in the next section to deduce the conjecture of Beauville and Catanese.

Let $K$ be a number field and let $A$ be an abelian variety defined over $K$. Let $X$ be a closed subvariety of $A$, which is defined over $K$. We do not assume that $X$ is irreducible. Let $U$ be an open subscheme of $\operatorname{Spec} \mathcal{O}_{K}$, such that $A$ extends to an abelian scheme $\mathcal{A}$ over $U$, and $X$ extends to a closed subscheme $\mathcal{X} \subset \mathcal{A}$.

Theorem 2.1. Suppose that for all but a finite number of primes $\mathfrak{p} \in U$ we have $p \cdot \mathcal{X}_{\overline{\mathfrak{p}}} \subseteq \mathcal{X}_{\overline{\mathfrak{p}}}$, where $p:=\operatorname{char}(k(\mathfrak{p}))$. Then $X$ is a finite union of translates of abelian subvarieties of $A$ by torsion points of $A(\bar{K})$.

The proof of Theorem 2.1 hinges on the following results.

\section{Results from algebraic geometry}

Let $A$ be an abelian variety over an algebraically closed field $L$, endowed with an isogeny $\varphi: A \rightarrow A$. We say that $A$ is pure of positive weight if there are positive integers $r$ and $s$, not necessarily relatively prime, such that $\varphi^{s}=$ Frob $_{p^{r}}$ for some model of $A$ over $\mathbb{F}_{p^{r}}$. We say that $A$ is pure of weight 0 if $\varphi$ is an automorphism of finite order on $A$. If $A$ is pure of either positive weight or pure of weight 0 , then we simply say that $A$ is pure. If $A^{\prime}$ is another abelian variety over $L$ endowed with an isogeny $\varphi^{\prime}$, we shall say that a homomorphism $f: A \rightarrow A^{\prime}$ is equivariant if $\varphi^{\prime} \circ f=f \circ \varphi$. The following theorem is proven in [10, Th. 3.1]. 
Theorem 2.2 (Pink-Roessler). Let A be an abelian variety over an algebraically closed field L, endowed with an isogeny $\varphi: A \rightarrow A$. Let $X \subset A$ be an irreducible, reduced closed subscheme satisfying $\varphi(X)=X$. Suppose that there is no non-trivial equivariant homomorphism from $A$ to a pure abelian variety. Then $X$ is linear.

Next, recall the following constructibility statements. Let $S$ be a noetherian scheme and $\pi: Y \rightarrow S$ a scheme of finite type over $S$. Recall Chevalley's theorem: the image by $\pi$ of a constructible set of $Y$ is constructible in $S$ (see [6, Th. 1.8.4, chap. IV, IHES no. 20, p. 239]).

Recall also that the following subsets of $S$ are constructible: $\left\{s: Y_{s}\right.$ is geometrically irreducible $\},\left\{s: Y_{s}\right.$ is geometrically reduced $\}$ (see [6, Th. 9.7.7, chap. IV, IHES 28, p. 79]).

Now suppose that $S$ is also integral and normal. Let $\eta \in S$ be its generic point and suppose that $k(\eta)$ is a field of characteristic 0 .

Proposition 2.3. Suppose that $Y$ is a closed subscheme of an abelian scheme $\mathcal{B}$ over $S$. If $Y_{\bar{s}}$ is linear in $\mathcal{B}_{\bar{s}}$ for all the $s \in D$, where $D$ is dense in $S$, then $Y_{\bar{\eta}}$ is linear.

Proof. Choose a finite extension $F$ of $k(\eta)$ where the irreducible components of $Y \times{ }_{S}$ Spec $F$ are geometrically irreducible. Let $f: S^{\prime} \rightarrow S$ be the normalisation of $S$ in $F^{\prime}$. We shall need the

Lemma 2.4. Let $g: X_{1} \rightarrow X_{2}$ be a surjective morphism of finite type, where $X_{1}$ and $X_{2}$ are integral noetherian schemes. Let $E \subseteq X_{2}$ be a dense subset of $X_{2}$. Then the set $g^{-1}(E)$ is dense in $X_{1}$.

Proof. Suppose that the conclusion does not hold and let $V \subseteq X_{1} \backslash g^{-1}(E)$ be a non-empty open subset of $X_{1}$. The set $g(V)$ is constructible by Chevalley's theorem and thus $\overline{g(V)} \neq X_{2}$ by hypothesis. The closed subset $g^{-1}(\overline{g(V)})$ is not equal to $X_{1}$ since $g$ is surjective. This contradicts the fact that $X_{1}$ is irreducible and finishes the proof.

By the lemma, the set $f^{-1}(D)$ is dense in $S^{\prime}$. Let $\pi^{\prime}: Y^{\prime} \rightarrow S^{\prime}$ be the scheme obtained from $Y$ by base-change. By the mentioned constructibility results and the hypothesis, there is an open set $V^{\prime} \subseteq S^{\prime}$ such that for each irreducible component $Z$ of $Y^{\prime} \cap\left(\pi^{\prime}\right)^{-1}\left(V^{\prime}\right), Z_{\bar{s}}$ is irreducible and reduced for all $s \in V^{\prime}$. Let $Z$ be any irreducible component of $Y^{\prime} \cap\left(\pi^{\prime}\right)^{-1}\left(V^{\prime}\right)$ and let $h, h^{\prime}, h^{\prime \prime} \in Z(F)$. If we further shrink $V^{\prime}$, we may suppose that $h, h^{\prime}, h^{\prime \prime}$ extend to sections $h, h^{\prime}, h^{\prime \prime} \in$ $Z\left(V^{\prime}\right)$. By hypothesis, we have $\left(h_{s}-h_{s}^{\prime \prime}\right)+\left(h_{s}^{\prime}-h_{s}^{\prime \prime}\right) \in Z_{s}(k(s))-h_{s}^{\prime \prime}$ for $s \in f^{-1}(D) \cap V^{\prime}$. Hence $\left(h-h^{\prime \prime}\right)+\left(h^{\prime}-h^{\prime \prime}\right) \in Z(F)-h^{\prime \prime}$. Similarly, we deduce that $-\left(h-h^{\prime \prime}\right) \in Z(F)-h^{\prime \prime}$. Since $F$ can be taken arbitrarily large, this implies that $\left(h-h^{\prime \prime}\right)+\left(h^{\prime}-h^{\prime \prime}\right) \in Z(\overline{k(\eta)})-h^{\prime \prime}$ and $-\left(h-h^{\prime \prime}\right) \in Z(\overline{k(\eta)})-h^{\prime \prime}$ for any $h, h^{\prime}, h^{\prime \prime} \in Z(\overline{k(\eta)})$. Thus $Z_{\overline{k(\eta)}}$ is linear and we are done. 


\section{Density results}

Theorem 2.5. For $1 \leq i \leq d$ let $A_{i}$ be an abelian variety over a number field $K$ and $a_{i} \in A_{i}(K)$ a rational point. Assume that for all finite places $v$ of $K$ in a set of Dirichlet density 1 the reduction of at least one $a_{i}$ is annihilated by a power of the residue characteristic $p_{v}$. Then at least one $a_{i}=0$.

Proof. This is [9, Th. 5.1]. The second author noticed that this also follows from results of Wong, Nori and Zarhin. See [9, Par. 5, Rem. 5.2] for details about this implication.

The following theorem is [9, Th. 5.3] and is more difficult to prove than Theorem 2.5 .

Theorem 2.6 (Pink). For $1 \leq i \leq d$ let $A_{i}$ be an abelian variety over a number field $K$ and $a_{i} \in A_{i}(K)$ a rational point. Let $f(T) \in \mathbb{Z}[T]$ be any polynomial which is a product of cyclotomic polynomials and a power of $T$. For any finite place $v$ of $K$ let $p_{v}$ denote the characteristic of the residue field and $a_{i, v}$ the reduction of $a_{i}$. Assume that for all finite places $v$ of $K$ in a set of Dirichlet density 1 at least one $a_{i, v}$ is annihilated by $f\left(p_{v}\right)$. Then at least one $a_{i}$ is a torsion point.

Finally we shall need a density statement on supersingular reduction. To state it consider any abelian variety $B$ over an algebraically closed field $L$ of characteristic $p>0$. Recall that $B$ is supersingular if and only if the Dieudonné module of its $p$-divisible group is pure of slope $1 / 2$. When $B$ possesses a model over a finite subfield $\mathbb{F}_{q} \subset L$ with $q$ elements, this is equivalent to saying that all the eigenvalues of the characteristic polynomial of the Frobenius morphism Frob $q$ have the form $\sqrt{q} \cdot z$, where $z$ is a root of unity. This in turn is equivalent to saying that $B$ is pure of positive weight for the isogeny given by multiplication by $p$. In general, $B$ is supersingular if and only if $B$ is isogenous to a supersingular abelian variety defined over a finite field.

We shall say that $B$ has a supersingular factor if it possesses a non-trivial subquotient which is a supersingular abelian variety. Since abelian varieties are semisimple up to isogeny, this is equivalent to saying that there exists a non-trivial homomorphism from $B$ to an abelian variety which is pure of positive weight for the isogeny given by multiplication by $p$. Note that multiplication by $p$ is never an automorphism of finite order on any non-trivial abelian variety over $L$. Thus saying that $B$ has no supersingular factor is equivalent to saying that there exists no nontrivial homomorphism from $B$ to an abelian variety which is pure for the isogeny given by multiplication by $p$, which is just the condition from Theorem 2.2.

Theorem 2.7 (Pink). Let $A$ be an abelian variety over a number field $K$. Then there exists a finite extension $L$ of $K$ such that for all finite places of $L$ in a set of Dirichlet density 1 the reduction of $A \times_{K} L$ does not have a supersingular factor.

For the proof see [9, Cor. 1.7]. 


\section{Proof of Theorem 2.1}

Recall that $U$ is an open subscheme of $\operatorname{Spec} \mathcal{O}_{K}$, such that $A$ extends to an abelian scheme $\mathcal{A}$ over $U$, and $X$ extends to a closed subscheme $\mathcal{X} \subset \mathcal{A}$. After shrinking $U$ we may suppose that $p \cdot \mathcal{X}_{\overline{\mathfrak{p}}} \subseteq \mathcal{X}_{\overline{\mathfrak{p}}}$ for all $U$.

Before beginning with the proof of Theorem 2.1, notice that one of the difficulties of the proof is the fact that we do not require $X$ to be geometrically irreducible. If $X$ is geometrically irreducible, then Theorem 2.7 together with Theorem 2.2 immediately imply that $\mathcal{X}_{\bar{p}}$ is linear for a set of places of density 1 and Proposition 2.3 implies that $X$ is linear. To show that $X$ is completely linear, just notice that the image of $X$ in $A / \operatorname{Stab}(X)$ is a point which is almost everywhere a $p-1$ torsion point. This implies that this point is a torsion point by Theorem 2.6 and concludes the proof in this case.

We shall now tackle the general case. We may suppose that the irreducible components $\mathcal{X}_{1}, \ldots, \mathcal{X}_{r}$ of $\mathcal{X}$ are flat over $U$ and that $X_{i}:=\mathcal{X}_{i, K}$ is defined over $K$ and geometrically irreducible for all $i \geq 1$. We may also suppose that $K$ is sufficiently large so that the conclusion of Theorem 2.7 holds for $\mathcal{A}$. Let $U_{0} \subseteq U$ be the set of non-archimedean places $\mathfrak{p}$ such that $\mathcal{X}_{1, \overline{\mathfrak{p}}}, \ldots \mathcal{X}_{r, \overline{\mathrm{p}}}$ are geometrically integral and such that $\mathcal{A}_{\bar{p}}$ has no supersingular factors. The set $U_{0}$ has Dirichlet density 1 by Theorem 2.7. Let $\mathfrak{p} \in U_{0}$. Consider the descending sequence

$$
\mathcal{X}_{\overline{\mathfrak{p}}} \supseteq p \cdot \mathcal{X}_{\overline{\mathfrak{p}}} \supseteq p^{2} \cdot \mathcal{X}_{\overline{\mathfrak{p}}} \supseteq \ldots
$$

where $p=\operatorname{char}(k(\mathfrak{p}))$. By Noethericity, this sequence must stabilize and we call $Y_{\overline{\mathfrak{p}}}$ the first stable term. By the construction of $Y_{\overline{\mathfrak{p}}}$ there exists $k_{0} \geq 0$ such that

$$
\mathcal{X}_{\overline{\mathfrak{p}}} \subseteq\left(p^{k_{0}}\right)^{-1} Y_{\overline{\mathfrak{p}}}
$$

Furthermore, Theorem 2.2 and the equality $p \cdot Y_{\overline{\mathfrak{p}}}=Y_{\overline{\mathfrak{p}}}$ implies that $Y_{\overline{\mathfrak{p}}}$ is linear. Fix $i \geq 1$. The inclusion (1) now implies that $\mathcal{X}_{i, \bar{p}}$ is contained in a closed set of the form $t+Z$ where $Z$ is a (necessarily linear) irreducible component of $Y_{\bar{p}}$ and $t$ is a $p^{k_{0}}$-torsion point of $\mathcal{A}_{\overline{\mathfrak{p}}}(\overline{k(\mathfrak{p})})$. Amongst the pairs $t, Z$ with this property, choose one such that the dimension of $Z$ is maximal. We claim: $Z$ is an irreducible component of $\mathcal{X}_{\overline{\mathfrak{p}}}$ as well. To see this, suppose the contrary, i.e. that there is an irreducible component $Z^{\prime}$ of $\mathcal{X}_{\bar{p}}$ such that $Z \subseteq Z^{\prime}$ and $Z \neq Z^{\prime}$. There exists $t^{\prime}$, a $p^{k_{0}}$-torsion point and an irreducible component $Z^{\prime \prime}$ of $Y_{\bar{p}}$ such that $Z^{\prime} \subseteq t^{\prime}+Z^{\prime \prime}$. This implies that there is an inclusion $\mathcal{X}_{i, \bar{p}} \subseteq\left(t+t^{\prime}\right)+Z^{\prime \prime}$, where $\operatorname{dim}\left(Z^{\prime \prime}\right)>\operatorname{dim}(Z)$, which is impossible, thus proving the claim. We deduce that there is a function

$$
j_{\overline{\mathfrak{p}}}:\{1, \ldots, r\} \rightarrow\{1, \ldots, r\}
$$

such that

$$
\mathcal{X}_{i, \bar{p}} \subseteq t_{i, \overline{\mathfrak{p}}}+\mathcal{X}_{j_{\overline{\mathfrak{p}}(i), \overline{\mathfrak{p}}}}
$$


where $\mathcal{X}_{j_{\overline{\mathfrak{p}}(i), \overline{\mathfrak{p}}}}$ is linear and $t_{i, \overline{\mathfrak{p}}}$ is a $p^{\infty}$-torsion point. Let now $J_{i}$ be the subset of $\{1, \ldots, r\}$ of elements that are equal to $j_{\overline{\mathfrak{p}}}(i)$ for an infinity of $\mathfrak{p} \in U_{0}$. Let $l \in J_{i}$. Proposition 2.3 implies that $X_{l}$ is linear and thus the image of $X_{l}$ in $A / \operatorname{Stab}\left(X_{l}\right)$ is a point $Q_{l}$. Similarly, the image of $\mathcal{X}_{i, \overline{\mathfrak{p}}}$ in $\mathcal{A}_{\overline{\mathfrak{p}}} / \operatorname{Stab}\left(\mathcal{X}_{l, \overline{\mathfrak{p}}}\right)$ is a point for an infinity of $\mathfrak{p} \in U$ and thus the image of $X_{i}$ in $A / \operatorname{Stab}\left(X_{l}\right)$ is also a point $P_{l}$. Consider the set of points $P_{l}-Q_{l}$, where $l \in J_{i}$. This set has the property that for all but a finite number of places $\mathfrak{p} \in U_{0}$, there is an $l \in J_{i}$ such that $\left(P_{l}-Q_{l}\right)(\bmod \mathfrak{p})$ is a $p^{\infty}$-torsion point. Thus Theorem 2.5 implies that $P_{l}=Q_{l}$ for some $l \in J_{i}$. This implies that $\mathcal{X}_{i, \overline{\mathfrak{p}}}=\mathcal{X}_{l, \overline{\mathfrak{p}}}$ for an infinity of $\mathfrak{p} \in U$ and thus $X_{i}=X_{l}$; hence $X_{i}$ is linear and since $i$ was arbitrary $X$ is linear.

To prove that $X$ is completely linear, suppose that it is not and let $C$ be an irreducible component of $X$ which is a translate of an abelian subvariety $B$ of $A$ such that $C / B$ is not a torsion point. Suppose that $C$ is of the largest possible dimension with this property and let $\mathcal{C}$ be the corresponding irreducible component of $\mathcal{X}$. Let $c_{0}$ be the number of irreducible components of dimension $\operatorname{dim}(C)$. Let $C_{1}, \ldots, C_{h}$ be the irreducible components of $X$ which are of dimension larger than $\operatorname{dim}(C)$ and let $\mathcal{C}_{1}, \ldots, \mathcal{C}_{h}$ be the corresponding components of $\mathcal{X}$. Let $b_{i}$ $(1 \leq i \leq h)$ be the order of the point which is the image of $C_{i}$ in $A / \operatorname{Stab}\left(C_{i}\right)$. Let $n:=b_{1} ! \cdots \ldots \cdots b_{h} ! \cdot\left(c_{0}+1\right)$ !.

For almost all $\mathfrak{p} \in U_{0}$, we have:

(1) $p^{n} \cdot \mathcal{C}_{i, \overline{\mathfrak{p}}}=\mathcal{C}_{i, \overline{\mathrm{p}}}(1 \leq i \leq h)$;

(2) either $p^{n} \cdot \mathcal{C}_{\overline{\mathfrak{p}}}$ is an irreducible component of $\mathcal{X}_{\overline{\mathfrak{p}}}$ or $p^{n} \cdot \mathcal{C}_{\overline{\mathfrak{p}}}$ is contained in one of the $\mathcal{C}_{i, \overline{\mathfrak{p}}}$, where $i$ depends on $\mathfrak{p}$.

If $p^{n} \cdot \mathcal{C}_{\overline{\mathfrak{p}}}$ is an irreducible component of $\mathcal{X}_{\bar{p}}$, then the closed sets

$$
\mathcal{C}_{\overline{\mathfrak{p}}}, p \cdot \mathcal{C}_{\overline{\mathfrak{p}}}, p^{2} \cdot \mathcal{C}_{\overline{\mathfrak{p}}}, \ldots, p^{c_{0}+1} \cdot \mathcal{C}_{\overline{\mathfrak{p}}}
$$

are also irreducible components of $\mathcal{X}_{\bar{p}}$. Hence there are natural numbers $k_{\mathfrak{p}}$ and $l_{\mathfrak{p}}$, with $1 \leq k_{\mathfrak{p}}, l_{\mathfrak{p}} \leq c_{0}$, such that $p^{l_{\mathfrak{p}}} \cdot\left(p^{k_{\mathfrak{p}}} \cdot \mathcal{C}_{\overline{\mathfrak{p}}}\right)=\left(p^{k_{\mathfrak{p}}} \cdot \mathcal{C}_{\overline{\mathfrak{p}}}\right)$. This implies that $p^{n} \cdot\left(p^{k_{\mathfrak{p}}} \cdot \mathcal{C}_{\overline{\mathfrak{p}}}\right)=p^{k_{\mathfrak{p}}} \cdot \mathcal{C}_{\overline{\mathfrak{p}}}$ and finally that $p^{n+c_{0}} \cdot \mathcal{C}_{\overline{\mathfrak{p}}}=p^{c_{0}} \mathcal{C}_{\overline{\mathfrak{p}}}$.

After a permutation of the numbering, we may assume that $\mathcal{C}_{1}, \ldots, \mathcal{C}_{r}$ are the components which appear in (2) for an infinite number of places $(r \leq h)$. Consider the following set of points $S$ : the image of $C$ in $A / \operatorname{Stab}(C)$, the image of $C-C_{i}$ in $A / \operatorname{Stab}\left(C_{i}\right)$ where $1 \leq i \leq r$. Then for almost all $\mathfrak{p} \in U_{0}$, there is a $P \in S$ such that $p^{n} \cdot\left(p^{n+c_{0}}-p^{c_{0}}\right)(P \bmod \mathfrak{p})=0$. By Theorem 2.6, one of the elements of $S$ is a torsion point. So let $T$ be a torsion point in $S$. Suppose first that $T \neq 0$. The restriction of the reduction map $A(K) \rightarrow \mathcal{A}_{\mathfrak{p}}(k(\mathfrak{p}))$ to the set of torsion points of order prime to $p$ is injective for all $\mathfrak{p} \in U_{0}$. Hence $T$ cannot satisfy the condition $p^{n} \cdot(T \bmod \mathfrak{p})=0$ for an infinite number of $\mathfrak{p} \in U$, thus it has to be the image of $C$ in $A / \operatorname{Stab}(C)$. This implies that $C$ is a translate of $\operatorname{Stab}(C)$ by a torsion point, which is a contradiction. Thus $T=0$; but this implies that either $C$ is a translate of $\operatorname{Stab}(C)$ by a torsion point or $C \subseteq C_{i}$ for some $i$ with $1 \leq i \leq h$. This is the contradiction which concludes the proof of the theorem. 
Remark. In the above proof, Theorem 2.6 is only needed to prove that $X$ is completely linear. To prove that $X$ is linear, the theorems 2.5 and 2.7 suffice.

\section{A new proof of the conjecture of Beauville and Catanese}

The conjecture of Beauville and Catanese is now a simple consequence of the classification theorem 2.1 and of a result of Deligne-Illusie originally used to give an algebraic proof of the Kodaira-Nakano vanishing theorem.

We shall use the following terminology. If $Y$ is a smooth and projective variety over a field $k$ and $\mathcal{L}$ is a line bundle over $Y$, we define

$$
h^{i, j}(Y, \mathcal{L}):=\operatorname{dim}_{k}\left(H^{i}\left(Y, \Omega_{Y}^{j} \otimes \mathcal{L}\right)\right),
$$

where $\Omega_{Y}$ is the sheaf of differentials of $Y$ over $k$ and

$$
h_{D}^{r}(Y, \mathcal{L})=h_{D}^{r}(\mathcal{L}):=\sum_{i, j \geq 0, i+j=r} h^{i, j}(Y, \mathcal{L}) .
$$

Furthermore, we define

$$
S_{m}^{r}(Y):=\left\{\mathcal{L} \in \operatorname{Pic}^{0}(Y)(\bar{k}) \mid h_{D}^{r}\left(Y_{\bar{k}}, \mathcal{L}\right) \geq m\right\}
$$

and

$$
S_{m}^{i, j}(Y):=\left\{\mathcal{L} \in \operatorname{Pic}^{0}(Y)(\bar{k}) \mid h^{i, j}\left(Y_{\bar{k}}, \mathcal{L}\right) \geq m\right\}
$$

for any $r, m, i, j \geq 0$. Here $\operatorname{Pic}^{0}(Y)$ is the neutral component of the Picard scheme of $Y$; its underlying reduced scheme is an abelian variety over $k$. The sets $S_{m}^{r}(Y)$ and $S_{m}^{i, j}(Y)$ are Zariski-closed by the semi-continuity theorem and we endow them with their reduced induced subscheme structure. About these sets, we have the following result.

Proposition 3.1. Every irreducible component of $S_{m}^{i, j}(Y)$ is an irreducible component of $S_{m^{\prime}}^{i+j}(Y)$ for some $m^{\prime} \geq m$.

Proof. Let $Z$ be an irreducible component of $S_{m}^{i, j}(Y)$. Then by semicontinuity there exists an open dense subset $V \subset Z$ such that for all pairs $\left(i^{\prime}, j^{\prime}\right)$ the value $h_{Z}^{i^{\prime}, j^{\prime}}:=h^{i^{\prime}, j^{\prime}}\left(Y_{\bar{k}}, \mathcal{L}\right)$ for $\mathcal{L} \in V(\bar{k})$ is independent of $\mathcal{L}$. Moreover, again by semicontinuity there exists an open neighbourhood $U \subset \operatorname{Pic}^{0}(Y)$ of $V$ such that for all $\left(i^{\prime}, j^{\prime}\right)$ we have $h^{i^{\prime}, j^{\prime}}\left(Y_{\bar{k}}, \mathcal{L}\right) \leq h_{Z}^{i^{\prime}, j^{\prime}}$ for all $\mathcal{L} \in(U \backslash V)(\bar{k})$. Furthermore, since $Z$ is an irreducible component of $S_{m}^{i, j}(Y)$, we can choose $U$ and $V$ such that the last inequality is strict for $\left(i^{\prime}, j^{\prime}\right)=(i, j)$. Summing up over all pairs $\left(i^{\prime}, j^{\prime}\right)$ with $i^{\prime}+j^{\prime}=i+j$ we deduce that $h_{D}^{i+j}\left(Y_{\bar{k}}, \mathcal{L}\right)$ takes some constant value $m^{\prime}$ for all $\mathcal{L} \in V(\bar{k})$, and that $h_{D}^{i+j}\left(Y_{\bar{k}}, \mathcal{L}\right)<m^{\prime}$ for all $\mathcal{L} \in(U \backslash V)(\bar{k})$. This shows that $V=U \cap S_{m^{\prime}}^{i+j}(Y)$; hence its closure $Z$ is an irreducible component of $S_{m^{\prime}}^{i+j}(Y)$, as desired. 
To formulate the result of Deligne and Illusie, suppose now that $k$ is perfect of characteristic $p>0$ and let $Y$ be a smooth and projective variety over $k$. Let $\mathcal{L}$ be a line bundle over $Y$.

Theorem 3.2 (Deligne-Illusie). Suppose that $Y$ admits a lifting to a flat scheme over the ring $w_{2}(k)$ of the Witt vectors of length 2 of $k$ and that $\operatorname{dim}(Y) \leq \operatorname{char}(k)$. Then the inequality

$$
h_{D}^{r}(\mathcal{L}) \leq h_{D}^{r}\left(Y, \mathcal{L}^{\otimes p}\right)
$$

holds for all $r \geq 0$.

For the proof see [3, Lemma 2.9, par. 3, p. 258].

Let now $Y$ be a smooth projective variety over $\overline{\mathbb{Q}}$.

Proposition 3.3. For any $r, m \geq 0$, the scheme $S_{m}^{r}(Y)$ is completely linear.

Proof. Choose a number field $K$ which is a field of definition for $Y$. We shall thus view $Y$ as a $K$-scheme. Let $\mathcal{Y}$ be a smooth an projective model of $Y$ over an open subscheme $U_{0}$ of $\mathcal{O}_{K}$. Shrink $U_{0}$ so that $\operatorname{Pic}^{0}(Y)$ has a model $\mathcal{A}$ over $U_{0}$ which is an abelian scheme. Suppose also that the universal line bundle on $Y \times \operatorname{Pic}^{0}(Y)$ extends to a line bundle $\mathcal{M}$ on $\mathcal{Y} \times_{U_{0}} \mathcal{A}$ which is also compatible with the group scheme structure of $\mathcal{A}$. Let $S_{m}^{r}(\mathcal{Y}):=\left\{s \in \mathcal{A} \mid h_{D}^{r}\left(\mathcal{Y}_{k(s)}, \mathcal{M}(s)\right) \geq m\right\}$. This set is again closed by the semicontinuity theorem; endow $S_{m}^{r}(\mathcal{Y})$ with its reduced induced subscheme structure. By construction, its set of $\overline{\mathbb{Q}}$-points is $S_{m}^{r}(Y)(\overline{\mathbb{Q}})$. Furthermore, for all the closed $\mathfrak{p} \in U_{0}$ which are unramified over $\mathbb{Q}$ and of residue field characteristic $\geq \operatorname{dim}(Y)$, we have $p \cdot S_{m}^{r}(\mathcal{Y})_{\overline{\mathfrak{p}}} \overline{(\overline{k(\mathfrak{p})})} \subseteq S_{m}^{r}(\mathcal{Y})_{\overline{\mathfrak{p}}}(\overline{k(\mathfrak{p})})$. Thus if we apply Theorem 2.1 to (a cofinite subset of) $U_{0}$ and $S_{m}^{r}(Y)$, we are done.

If we combine the proposition 3.3 with the Proposition 3.1, we obtain

Corollary 3.4. For any $i, j, m \geq 0$, the scheme $S_{m}^{i, j}(Y)$ is completely linear.

Finally this implies the conjecture of Beauville and Catanese. We shall need the following lemma:

Proposition 3.5. Let $Y$ be a smooth projective variety over $\overline{\mathbb{Q}}$. Let $n \geq 1, r \geq 0$ and let $\mathcal{L}$ be a line bundle over $Y$, such that $\mathcal{L}^{\otimes n} \simeq \mathcal{O}_{Y}$. Then $h_{D}^{r}\left(\mathcal{L}^{\otimes k}\right)=h_{D}^{r}(\mathcal{L})$ if $(k, n)=1$.

Proof. Let $K$ be a number field which a field of definition for $Y$ and $\mathcal{L}$. Let $U \subset$ Spec $\mathcal{O}_{K}$ be an open subscheme of Spec $\mathcal{O}_{K}$ with the following properties:

- $Y$ has smooth and proper model $\mathcal{Y}$ over $U$;

- $\mathcal{L}$ extends to a line bundle $\widetilde{\mathcal{L}}$ over $\mathcal{Y}$ for which the identity $\widetilde{\mathcal{L}}^{\otimes n} \simeq \mathcal{O}_{\mathcal{Y}}$ also holds;

- all the non-archimedean places in $U$ are coprime to $n$, unramified over $\mathbb{Q}$ and of residue field characteristic $\geq \operatorname{dim}(Y)$. 
Let $\mathfrak{p}$ be any non-archimedean place in $U$. Let $l$ be the smallest integer such that $p^{l}=1(\bmod n)$, where $p=\operatorname{char}(k(\mathfrak{p}))$. By Theorem 3.2, we have

$$
h_{D}^{r}\left(\widetilde{\mathcal{L}}_{\mathfrak{p}}\right) \leq h_{D}^{r}\left(\widetilde{\mathcal{L}}_{\mathfrak{p}}^{\otimes p}\right) \leq h_{D}^{r}\left(\widetilde{\mathcal{L}}_{\mathfrak{p}}^{\otimes p^{l}}\right)=h_{D}^{r}\left(\widetilde{\mathcal{L}}_{\mathfrak{p}}\right)
$$

and thus $h_{D}^{r}\left(\widetilde{\mathcal{L}}_{\mathfrak{p}}\right)=h_{D}^{r}\left(\widetilde{\mathcal{L}}_{\mathfrak{p}}^{\otimes p}\right)$. By Dirichlet's theorem on arithmetic progressions, there is an infinity of prime numbers in the sequence $a \cdot n+k, a \rightarrow \infty$. Thus there is an infinity of non-archimedean places $\mathfrak{p}$ in $U$ such that

$$
h_{D}^{r}\left(\widetilde{\mathcal{L}}_{\mathfrak{p}}^{\otimes k}\right)=h_{D}^{r}\left(\widetilde{\mathcal{L}}_{\mathfrak{p}}^{\otimes(a \cdot n+k)}\right)=h_{D}^{r}\left(\widetilde{\mathcal{L}}_{\mathfrak{p}}^{\otimes p}\right)=h_{D}^{r}\left(\widetilde{\mathcal{L}}_{\mathfrak{p}}\right),
$$

where $a$ depends on $\mathfrak{p}$. Thus $h_{D}^{r}\left(\mathcal{L}^{\otimes k}\right)=h_{D}^{r}(\mathcal{L})$ and we are done.

Remark. The last lemma can also be proven by an analytic method.

Notice that Lemma 3.5 together with Proposition 3.1 immediately imply Proposition 1.2 in the case where $Y$ is a smooth and projective variety over $\overline{\mathbb{Q}}$.

Theorem 3.6. Let $Y$ be smooth projective variety over $\mathbb{C}$. Then the sets $S_{m}^{i, j}(Y)$ are completely linear. Furthermore Proposition 1.2 holds.

Proof. Let $L_{0}$ be a field of definition for $Y$, which is finitely generated over $\mathbb{Q}$. From now on, we shall view $Y$ as an $L_{0}$-variety. Choose a normal, integral scheme $S_{0}$ of finite type over $\mathbb{Q}$ so that the variety $Y$ can be extended to a smooth and projective fibration $\widetilde{f}: \widetilde{Y} \rightarrow S_{0}$. Suppose also that there is a section $S_{0} \rightarrow \widetilde{Y}$ and that the natural map $\mathcal{O}_{S_{0}} \rightarrow \widetilde{f}_{*} \mathcal{O}_{\widetilde{Y}}$ is universally an isomorphism (i.e. remains so after any base-change). This can be achieved by shrinking $S_{0}$. Let $\operatorname{Pic}(\widetilde{Y}) / S_{0}$ be the Picard scheme of $\widetilde{Y} / S_{0}$. There is a line bundle $\mathcal{M}$ on $\widetilde{Y} \times{ }_{S_{0}} \operatorname{Pic}(\widetilde{Y})$ with the obvious universal property with respect to line bundles which are rigidified along the section $S_{0} \rightarrow \widetilde{Y}$ (see [1, Prop. 4, chap. 8.2, p. 211]). There is an open and closed $S_{0}$-group-subscheme $\operatorname{Pic}^{\tau}(\widetilde{Y}) / S_{0}$ of $\operatorname{Pic}^{0}(\widetilde{Y})$ which is projective over $S_{0}$ and has the following property: for any point $s \in S_{0}$, the set $\operatorname{Pic}^{\tau}(\widetilde{Y})_{\bar{s}}(\overline{k(s)})$ coincides with the line bundles on $\widetilde{Y}_{\bar{S}}$ whose image in the Neron-Severi group of $\widetilde{Y}_{\bar{S}}$ is of finite order (see [1, Th. 3, Th. 4, chap. 8.4]). The reduced identity component of $\operatorname{Pic}^{\tau}(\widetilde{Y})_{\bar{s}}$ coincides with the Picard variety $\operatorname{Pic}^{0}\left(\widetilde{Y}_{\bar{s}}\right)$. As $\operatorname{Pic}^{\tau}\left(Y_{\overline{L_{0}}}\right)$ is smooth, we may replace $S_{0}$ by one of its open subsets and suppose that $\operatorname{Pic}^{\tau}(\widetilde{Y})$ is smooth over $S_{0}$. After replacing $S_{0}$ by its normalisation in a finite extension and further shrinking $S_{0}$, we may suppose that there is an irreducible component $\operatorname{Pic}^{0}(\widetilde{Y})$ of $\operatorname{Pic}^{\tau}(\widetilde{Y})$ such that $\operatorname{Pic}^{0}(\widetilde{Y})_{\bar{s}}=\operatorname{Pic}^{0}\left(\widetilde{Y}_{\bar{s}}\right)$ for all $s \in S_{0}$. Let

$$
S_{m}^{i, j}(\widetilde{Y}):=\left\{s \in \operatorname{Pic}^{0}(\widetilde{Y}) \mid \operatorname{dim}_{k(s)} H^{i}\left(\widetilde{Y}_{s}, \Omega_{\widetilde{Y}_{s}}^{j} \otimes \mathcal{M}(s)\right) \geq m\right\}
$$

which is a Zariski-closed set. Endow this set with its reduced-induced subscheme structure. For each closed point $s \in S_{0}$, the residue field $k(s)$ is a number field and thus $S_{m}^{i, j}\left(\widetilde{Y}_{\bar{S}}\right)$ is completely linear by Proposition 3.3. Furthermore, closed 
points are dense in $S_{0}$ and by construction $S_{m}^{i, j}(\tilde{Y})\left(\overline{L_{0}}\right)=S_{m}^{i, j}(Y)\left(\overline{L_{0}}\right)$. Thus, using Proposition 2.3, we conclude that $S_{m}^{i, j}\left(Y_{\overline{L_{0}}}\right)$ is linear.

Now consider that since $S_{m}^{i, j}\left(\widetilde{Y}_{\bar{s}}\right)$ is completely linear, there exists a constant $c_{s} \geq 2$ such that $c_{s} \cdot S_{m}^{i, j}\left(\widetilde{Y}_{\bar{s}}\right)=S_{m}^{i, j}\left(\widetilde{Y}_{\bar{s}}\right)$ for each closed point $s$ in $S_{0}$. We may choose $c_{s}:=l_{s}^{\prod_{t} \varphi\left(d_{t, s}\right)}$. Here $d_{t, s}$ is the order of the image of $C_{t, s}$ in $\operatorname{Pic}^{0}\left(\widetilde{Y}_{\bar{s}}\right) / \operatorname{Stab}\left(C_{t, s}\right)$, where $C_{t, s}$ runs through the irreducible components of $S_{m}^{i, j}\left(\widetilde{Y}_{\bar{s}}\right)$; the number $l_{s}$ is the smallest prime number wich is larger than $d_{t, s}$ for all $t$. Now further shrink $S_{0}$ so that for all $m^{\prime} \geq m$, the number of irreducible components of $S_{m^{\prime}}^{i+j}\left(\widetilde{Y}_{\bar{S}}\right)$ remains constant. By Lemma 3.5 and Proposition 3.1, the numbers $l_{s}$ and $d_{t, s}$ have to remain bounded on $S_{0}$ (otherwise the number of irreducible components of $\cup_{m^{\prime} \geq m} S_{m,,^{\prime}}^{i+j}\left(\widetilde{Y}_{\bar{S}}\right)$ couldn't be finite). Thus $c_{s}$ remains bounded too. This implies that there is a dense subset $D \subseteq S_{0}$ such that $c_{s}$ is a constant $c_{0}$ in $D$. This in turn implies that $c_{0} \cdot S_{m}^{i, j}\left(Y_{\overline{L_{0}}}\right)=\overline{S_{m}^{i, j}}\left(Y_{\overline{L_{0}}}\right)$ and thus that $S_{m}^{i, j}\left(Y_{\overline{L_{0}}}\right)$ is completely linear. The estimate of Proposition 1.2 then follows from the corresponding estimate for $S_{m}^{i, j}\left(\widetilde{Y}_{\bar{S}}\right)$, where $s$ varies in a dense subset of closed points of $S_{0}$.

Remark. One could also have used Hilberts irreducibility theorem (see [4, Cor. 6.3, p. 244]) instead of Proposition 3.5 to prove that $S_{m}^{i, j}\left(Y_{\overline{L_{0}}}\right)$ is completely linear.

\section{The conjecture of Beauville and Catanese over perfect fields of positive characteristic}

The proof given in section 3 of the conjecture of Beauville and Catanese combines results in positive characteristic with density results. In this section, we shall briefly indicate the consequences of the positive characteristic results alone for the natural analog of the conjecture of Beauville and Catanese over a perfect field of positive characteristic.

Proposition 4.1. Let $Y$ be a smooth and projective variety over a perfect field $k$ of characteristic $p>0$. Suppose that $Y$ admits a flat lift to a scheme over $w_{2}(k)$ and that $\operatorname{dim}(Y) \leq \operatorname{char}(k)$. Suppose furthermore that the Picard variety of $Y$ has no supersingular factors. Then for any integers $r, m \geq 0$, the irreducible components of maximal dimension of $S_{m}^{r}(Y)$ are completely linear.

Proof. By Theorem 3.2, we have $p \cdot S_{m}^{r}(Y) \subseteq S_{m}^{r}(Y)$. Let $C$ be an irreducible component of maximal dimension of $S_{m}^{r}(Y)$. Since the multiplication by $p$ map is finite, $p^{k} \cdot C$ is also an irreducible component of maximal dimension, for all $k \geq 1$. Since the number of such components is finite, there are integers $k_{2}>k_{1} \geq 1$ such that $p^{k_{1}} \cdot C=p^{k_{2}} \cdot C$. Let $C^{\prime}:=p^{k_{1}} \cdot C$. Since $p^{k_{2}-k_{1}} \cdot C^{\prime}=C^{\prime}$, the Theorem 2.2 implies that $C^{\prime}$ is completely linear. Since $C \subseteq\left(p^{k_{1}}\right)^{-1}\left(C^{\prime}\right)$, we deduce that $C$ is also completely linear. 
If we combine the last proposition with the Proposition 3.1, we obtain

Corollary 4.2. Let $Y$ be a smooth and projective variety defined over a finite field $k$. Suppose that $Y$ admits a flat lift to a scheme over $w_{2}(k)$ and that $\operatorname{dim}(Y) \leq$ char $(k)$. Suppose furthermore that the Picard variety of $Y$ is simple and not supersingular. Then for any integers $i, j, m \geq 0$, the set $S_{m}^{i, j}(Y)$ is finite.

Another noteworthy result is

Proposition 4.3. Let $Y$ be a smooth and projective variety defined over a finite field $k$. Suppose that $Y$ admits a flat lift to a scheme over $w_{2}(k)$ and that $\operatorname{dim}(Y) \leq$ $p:=\operatorname{char}(k)$. Suppose furthermore that the Picard variety of $Y$ has no supersingular factors and has p-rank 0 . Then for any integers $i, j, m \geq 0$, the scheme $S_{m}^{i, j}(Y)$ is completely linear.

Proof. The condition on the $p$-rank insures that $p \cdot S_{m}^{r}(Y)=S_{m}^{r}(Y)$. This follows from the fact that all the $\bar{k}$-points of $S_{m}^{r}(Y)$ are then torsion points of order prime to $p$. We can thus apply Theorem 2.2 to conclude that $S_{m}^{r}(Y)$ is linear and Proposition 3.1 to conclude the proof.

\section{A conjecture}

We shall formulate a conjecture which is suggested by Proposition 3.5.

Conjecture 5.1. Let $X$ be a smooth and projective variety over a perfect field $L$ of positive characteristic. Suppose that $X$ has a flat lift to $w_{2}(L)$ and that $\operatorname{dim}(X) \leq \operatorname{char}(L)$. Let $\mathcal{L}$ be a line bundle on $X$ which is defined over L. Fix $n \geq 1$ and suppose that $\mathcal{L}^{\otimes n} \simeq \mathcal{O}_{X}$. Then

$$
h_{D}^{r}(X, \mathcal{L})=h_{D}^{r}\left(X, \mathcal{L}^{\otimes k}\right)
$$

for all $k \geq 1$ such that $(k, n)=1$.

The theorem 3.2 shows that this conjecture holds when $L$ has positive characteristic $p$ and $k=p$. The Proposition 3.5 shows that the natural characteristic 0 analog of the conjecture holds.

A positive answer to the latter conjecture would imply that the natural positive characteristic analog of the conjecture of Beauville and Catanese holds for $X$. To show this implication, one needs the Weil conjectures and an easy special case of Theorem 2.2. 


\section{Function fields}

In this section, we shall tackle the function field analog of the sections 2 and 3 . The analog of section 2 is simpler and relies on earlier results by Hrushovski.

Linear varieties. We want to formulate and prove the analog of Theorem 2.1 (Theorem 6.1) when $K$ is a function field in one variable over a number field $k$. Let $U \subseteq$ Spec $\mathcal{O}_{k}$ be an open subset and let $S$ be an affine smooth (relative) curve over $U$, whose field of rational functions is $K$. Let $\mathcal{A} \rightarrow S$ be an abelian scheme and let $A:=\mathcal{A} \times{ }_{S} K$. We suppose that there are no non-trivial $\bar{K}$-homomorphisms from an abelian variety which has a model over a number field to $A$. This is by definition equivalent to saying that $A$ has no isotrivial factors. For any point $\mathfrak{p} \in U$, denote by $S_{\mathfrak{p}}$ the affine curve over $k(\mathfrak{p})$ obtained by base-change. We also write $K_{\mathfrak{p}}$ for the function-field of $S_{\mathfrak{p}}$. If $Y / S$ is an $S$-scheme, we then write $Y_{\mathfrak{p}}$ for the $S_{\mathfrak{p}}$-scheme $Y_{\mathfrak{p}} / S_{\mathfrak{p}}$ obtained by base-change, $Y_{K_{\mathfrak{p}}}$ for the corresponding scheme over Spec $K_{\mathfrak{p}}$ and $Y_{\overline{K_{\mathfrak{p}}}}$ for the corresponding scheme over Spec $\overline{K_{\mathfrak{p}}}$. The aim is to prove

Proposition 6.1. Let $\mathcal{X} \hookrightarrow \mathcal{A}$ be a closed $S$-subscheme such that $X:=\mathcal{X} \times{ }_{S} \bar{K}$ is reduced. Suppose that it has the following property: for any closed point $\mathfrak{p} \in U$, p. $\mathcal{X}_{\overline{K_{\mathfrak{p}}}} \subseteq \mathcal{X}_{\overline{K_{\mathfrak{p}}}}$, where $p=\operatorname{char}(k(\mathfrak{p}))$. Then $Y_{\bar{K}}$ is completely linear.

Proof. The proof hinges on the following facts, all proven by Hrushovski in [7]:

(*) Let $Y$ be a geometrically reduced, geometrically irreducible closed $K_{\mathfrak{p}}$ subscheme of dimension $\geq 1$ of $\mathcal{A}_{K_{\mathfrak{p}}}$, where $\mathfrak{p} \in U$ is closed and suppose that the stabiliser of $Y$ in $\mathcal{A}_{K_{\mathfrak{p}}}$ is finite. If for all $k \geq 0, p^{k} . \mathcal{A}_{K_{\mathfrak{p}}}\left(\left(K_{\mathfrak{p}}\right)^{\text {sep }}\right) \cap Y\left(\overline{K_{\mathfrak{p}}}\right)$ is geometrically dense in $Y$, then there is a non-trivial $\overline{K_{\mathfrak{p}}}$-map from an abelian variety defined over a finite field to $\mathcal{A}_{\overline{K_{\mathfrak{p}}}}$ (more is true; see [2, th. 3.3, p. 192]). Here $\left(K_{\mathfrak{p}}\right)^{\text {sep }} \subseteq \overline{K_{\mathfrak{p}}}$ is the separable closure of $K_{\mathfrak{p}}$.

Remark. The only known proof of $(*)$ is via model theory.

(**) For almost all $\mathfrak{p} \in U$, the reduction map $A(K) \rightarrow \mathcal{A}_{K_{\mathfrak{p}}}\left(K_{\mathfrak{p}}\right)$ is injective (see [7, Lemma 4, p. 199]).

$(* * *)$ For almost all $\mathfrak{p} \in U$, there are no non-trivial $\overline{K_{\mathfrak{p}}}$-maps from an abelian variety defined over a finite field to $\mathcal{A}_{\overline{K_{\mathfrak{p}}}}$ (see [7, Cor. 8, p. 201]).

(*)' Let $Y$ be a geometrically reduced, geometrically irreducible closed $K_{\mathfrak{p}}$ subscheme of $\mathcal{A}_{K_{\mathfrak{p}}}$, where $\mathfrak{p} \in U$ is closed. Suppose that for all $k \geq 0, p^{k} . \mathcal{A}_{K_{\mathfrak{p}}}$ $\left(\left(K_{\mathfrak{p}}\right)^{\text {sep }}\right) \cap Y\left(\overline{K_{\mathfrak{p}}}\right)$ is geometrically dense in $Y$. Furthermore suppose that there are no non-trivial $\overline{K_{\mathfrak{p}}}$-maps from an abelian variety defined over a finite field to $\mathcal{A}_{\overline{K_{\mathfrak{p}}}}$. Then $Y$ must be the translate of an abelian subvariety.

The statement (*)' follows from the statement (*), applied to the quotient of $\mathcal{A}_{K_{\mathfrak{p}}}$ by the stabilisor of $Y$. We shall now prove: 


\section{(1) $X$ is linear.}

For this, it is sufficient to prove that $\mathcal{X}_{\overline{K_{\mathfrak{p}}}}$ is linear for almost all $\mathfrak{p} \in U$. We may replace $K$ by a finite extension and remove a finite number of closed points from $U$ if necessary. Thus we may assume that, for any $\mathfrak{p} \in U$, the base change to $\overline{K_{\mathfrak{p}}}$ of an irreducible component of $\mathcal{X}$ is irreducible, reduced and of the same dimension; we may also assume that the irreducible components of $\mathcal{X}$ remain distinct after base change to $\overline{K_{\mathfrak{p}}}$. We may also assume that (***) holds for all $\mathfrak{p} \in U$.

As in the proof of Theorem 2.1, for some fixed $\mathfrak{p} \in U$, we consider the descending sequence

$$
\mathcal{X}_{\overline{K_{\mathfrak{p}}}} \supseteq p \cdot \mathcal{X}_{\overline{K_{\mathfrak{p}}}} \supseteq p^{2} \cdot \mathcal{X}_{\overline{K_{\mathfrak{p}}}} \supseteq \ldots
$$

which must stabilize by Noethericity and we denote by $Z(\mathfrak{p})$ the first stable term. Fact (*) (perhaps applied to an extension of $K_{\mathfrak{p}}$ ) and (***) imply that $Z(\mathfrak{p})$ is linear. The theorem 2.2 has the same consequence and can be used as a substitute for $(*)$ and $(* * *)$. Therefore each irreducible component of $\mathcal{X}_{\overline{K_{\mathfrak{p}}}}$ is contained in an irreducible component of $Z(\mathfrak{p})$ translated by an element of $\mathcal{A}_{\overline{K_{\mathfrak{p}}}}\left[p^{\infty}\right]$, i.e. a torsion point of order a power of $p$ in $\mathcal{A}_{\overline{K_{\mathfrak{p}}}}\left(\overline{K_{\mathfrak{p}}}\right)$. Let $W_{0}$ be an irreducible component of $\mathcal{X}_{\overline{K_{\mathfrak{p}}}}$; we know that $W_{0} \subseteq B(\mathfrak{p})+P(\mathfrak{p})$, where $B(\mathfrak{p})$ is a linear irreducible component of $Z(\mathfrak{p})$ and $P(\mathfrak{p}) \in \mathcal{A}_{\overline{K_{\mathfrak{p}}}}\left[p^{\infty}\right]$; choose $B(\mathfrak{p})$ of maximal dimension with this property. As in the proof of Theorem $2.1, B(\mathfrak{p})$ is then an irreducible component of $\mathcal{X}_{\overline{K_{\mathfrak{p}}}}$. Let $\widetilde{W}_{0}$ be the irreducible component of $X$ corresponding to $W_{0}$. Since $U$ is infinite and the number of irreducible components of $X$ is finite, we see that there exists a linear irreducible component $\widetilde{B}$ of $X$ corresponding to $B(\mathfrak{p})$ for an infinite number of places $\mathfrak{p} \in U$. Consider now the image of $\widetilde{W}_{0}$ in the quotient $A / \widetilde{B}$. This is a point $Q \in A / \widetilde{B}(K)$, with the property that, for an infinite number of places $\mathfrak{p} \in U$, its reduction mod. $K_{\mathfrak{p}}$ is a $p^{\infty}$-torsion point. Fact $(* *)$ (applied to $A / \widetilde{B}$ rather than $A$ ) then implies that $Q=0$. Thus $X$ is linear.

(2) $X$ is completely linear.

We first shrink $U$ even further so that for all $\mathfrak{p} \in U, \mathcal{X}_{\overline{K_{\mathfrak{p}}}}$ is linear. For any $\mathfrak{p} \in U$ and any $d \geq 0$, let $\mathcal{X}_{\overline{K_{\mathfrak{p}}}, d}$ be the union of the irreducible components of dimension $d$ of $\mathcal{X}_{\overline{K_{\mathfrak{p}}}}$.

First, we claim that for any $d \geq 0$ the following holds: for almost all closed $\mathfrak{p} \in U, p \cdot \mathcal{X}_{\overline{K_{\mathfrak{p}}}, d} \subseteq \mathcal{X}_{\overline{K_{\mathfrak{p}}}, d}$.

To prove the claim, suppose the contrary. This implies that there is an infinite set $I_{0} \subseteq U$, such that for all $\mathfrak{p} \in I_{0}$, there exists an irreducible component $C_{\mathfrak{p}}$ of $\mathcal{X}_{\overline{K_{\mathfrak{p}}}}$, such that $p \cdot C_{\mathfrak{p}}$ is contained in an irreducible component $D_{\mathfrak{p}}$ of $\mathcal{X}_{\overline{K_{\mathfrak{p}}}}$, such that $\operatorname{dim}\left(D_{\mathfrak{p}}\right)>\operatorname{dim}\left(C_{\mathfrak{p}}\right)$. For each $\mathfrak{p} \in I_{0}$, choose $C_{\mathfrak{p}}$ of maximal dimension with this property. Let $C$ be an irreducible component of $X$ which corresponds to $C_{\mathfrak{p}}$ for all $\mathfrak{p}$ in an infinite set $I_{1} \subseteq I_{0}$. Let $D$ be an irreducible component of $X$ which corresponds to $D_{\mathfrak{p}}$ for all $\mathfrak{p}$ in an infinite set $I_{2}$ contained in $I_{1}$. For all $\mathfrak{p} \in I_{2}$, we then have $C_{\mathfrak{p}} \subseteq D_{\mathfrak{p}}^{\prime}+l_{\mathfrak{p}}$, where $D_{\mathfrak{p}}^{\prime}$ is an irreducible component of $\mathcal{X}_{\overline{K_{\mathfrak{p}}}}$ which is of dimension $\operatorname{dim}(D)$ and $l_{\mathfrak{p}}$ is a torsion point whose order is a 
power of $p$. Let $D^{\prime}$ be an irreducible component of $X$ which corresponds to $D_{\mathfrak{p}}^{\prime}$ in an infinite set $I_{3} \subseteq I_{2}$. The variety $C / \operatorname{Stab}\left(D^{\prime}\right)$ is then a non-zero point, whose reduction is of order $p$ for an infinite number of $\mathfrak{p}$, which is a contradiction to $(* *)$.

Secondly, we claim: for almost all closed $\mathfrak{p} \in U, \mathcal{X}_{\overline{K_{\mathfrak{p}}}}$ is completely linear.

To prove the claim, fix a $\mathfrak{p} \in U$ such that for all $d \geq 0, p \cdot \mathcal{X}_{\overline{K_{\mathfrak{p}}}, d} \subseteq \mathcal{X}_{\overline{K_{\mathfrak{p}}}, d}$. Let $C:=t(\mathfrak{p})+B(\mathfrak{p})$ be any irreducible component of $\mathcal{X}_{\overline{K_{\mathfrak{p}}}}$. Here $B(\mathfrak{p})$ is an abelian subvariety of $\mathcal{A}_{\overline{K_{\mathfrak{p}}}}$ and $t(\mathfrak{p}) \in \mathcal{A}_{\overline{K_{\mathfrak{p}}}}\left(\overline{K_{\mathfrak{p}}}\right)$. Let $c_{0}$ be the number of irreducible components of dimension $\operatorname{dim}(B(\mathfrak{p}))$ of $\mathcal{X}_{\mathfrak{p}}$ (and thus of $X$ ). As in the proof of 2.1 , we deduce that $p^{c_{0} !} p^{c_{0}} \cdot C=p^{c_{0}} \cdot C$. The image of $t(\mathfrak{p})$ in $\mathcal{A}_{\overline{K_{\mathfrak{p}}}} / B(\mathfrak{p})$ is thus in the kernel of $\left(p^{c_{0} !}-1\right) p^{c_{0}}$ and is thus a torsion point. Consider now any irreducible component $t+B$ of $X$, where $t \in A(K)$ and $B$ is an abelian subvariety of $A$. By the first claim and the second claim together, the image of $t$ in $A / B$ is a point in $(A / B)(K)$, such that for almost all closed $\mathfrak{p} \in U$, its reduction mod. $K_{\mathfrak{p}}$ is a torsion point. Fact $(* *)$ implies that $t$ is torsion point. This concludes the proof.

The conjecture of Beauville and Catanese. The Theorem 6.1 can be combined with a relative generalisation of 3.2 (relying on [3, Cor. 3.7, p. 263]) to obtain the following theorem, which is the function field case of the conjecture of Beauville and Catanese.

Theorem 6.2. Let $Y$ be a smooth projective variety defined over a function field $K$ of characteristic 0 . Suppose that $\operatorname{Pic}^{0}\left(Y_{\bar{K}}\right)$ has no isotrivial factors. Then the sets $S_{m}^{i, j}(Y)$ are completely linear for all integers $i, j, m \geq 0$.

Since this theorem can reduced to a special case of Theorem 3.6 using the Lefschetz principle, we shall not give the details of the proof.

\section{References}

[1] Bosch, S.; Lütkebohmert, W., Raynaud, M.: Néron models. Ergebnisse der Mathematik und ihrer Grenzgebiete (3), 21 Springer-Verlag, Berlin, 1990

[2] Bouscaren, E.: Proof of the Mordell-Lang conjecture for function fields. In: Model theory and algebraic geometry. An introduction to E. Hrushovski's proof of the geometric Mordell-Lang conjecture. Edited by Elisabeth Bouscaren. Lecture Notes in Mathematics, 1696. Springer-Verlag, Berlin, 1998

[3] Deligne, P., Illusie, L.: Relèvements modulo $p^{2}$ et décomposition du complexe de de Rham. Invent. Math. 89 (2), 247-270 (1987)

[4] Lang, S.: Fundamentals of Diophantine geometry. Springer-Verlag, New York, 1983

[5] Green, M., Lazarsfeld, R.: Higher obstructions to deforming cohomology groups of line bundles. J. Amer. Math. Soc. 4 (1), 87-103 (1991)

[6] Grothendieck, A., Dieudonné, J.: Éléments de géométrie algébrique. Publ. Math. Inst. Hautes Etud. Sci. 
[7] Hrushovski, E.: Proof of Manin's theorem by reduction to positive characteristic. In: Model theory and algebraic geometry. An introduction to E. Hrushovski's proof of the geometric Mordell-Lang conjecture. Edited by Elisabeth Bouscaren. Lecture Notes in Mathematics, 1696. Springer-Verlag, Berlin, 1998

[8] Nori, M.V.: On subgroups of $\mathrm{GL}_{n}\left(\mathbb{F}_{p}\right)$. Invent. Math. 88, 257-275 (1987)

[9] Pink, R.: On the Order of the Reduction of a Point on an Abelian Variety. Math. Ann. (2004) DOI: 10.1007/s00208-004-0548-8

[10] Pink, R., Roessler, D.: On psi-invariant subvarieties of semiabelian varieties and the ManinMumford conjecture. To appear in the Journal of Algebraic Geometry

[11] Simpson, C.: Subspaces of moduli spaces of rank one local systems. Ann. Sci. École Norm. Sup. (4) 26 (3), 361-401 (1993)

[12] Wong, S.: Power residues on abelian varieties. Manuscripta Math. 102 (1), 129-138 (2000)

[13] Zarhin, Y.: A finiteness theorem for unpolarized Abelian varieties over number fields with prescribed places of bad reduction. Invent. Math. 79, 309-321 (1985) 